Pramāna, Vol. 18, No. 3, March 1982, pp. 237-241. (C) Printed in India.

\title{
On the non-existence of hydrodynamic solution of surface plasmons at the bimetallic interface
}

\author{
C UBEROI and J REYES* \\ Department of Applied Mathematics, Indian Institute of Science, \\ Bangalore 560 012, India \\ *Instituto de Ciencias de la Universidad, \\ Autonoma de Puebla, Apdo. Postal J-48, Puebla, Pue. Mexico \\ MS received 30 October 1981; revised 31 December 1981
}

\begin{abstract}
It is shown that for an abrupt bimetallic interface a hydrodynamic solution for interface plasmons does not exist. It appears that this result is valid irrespective of the choice of of the additional boundary condition, thereby suggesting a careful look at the use of usual hydrodynamic equations for a bimetallic interface.
\end{abstract}

Keywords. Surface plasmons; bimetallic interface; hydrodynamic solution.

\section{Introduction}

It is well-known (Stern and Ferrel 1960; Kunz 1966) that a bimetallic interface supports surface plasmons with frequency $\omega_{s I}=\left(\frac{\omega_{p 1}^{2}+\omega_{p 2}^{2}}{2}\right)^{1 / 2}$ where $\omega_{p 1,2}$ are plasma frequencies of the two media. This result is obtained by using a local dielectric model for the electron gas. It is also well established that when hydrodynamic model is used, the surface plasmons along a metal-vacuum interface become dispersive and the frequency $\omega_{s V}$ is given as (Fuchs and Kliewer 1971)

$$
\omega_{s} V=\frac{\omega_{p 1}}{\sqrt{2}}\left[1+\left(\frac{3}{10}\right)^{1 / 2} v \frac{l}{\omega_{p 1}}+\ldots\right],
$$

where $v_{F}$ is the Fermi velocity. Hence, using the usual (Fuchs and Kliewer 1971) hydrodynamic model when instead of metal-vacuum interface a metal-metal interface is chosen, one expects a dispersion of the surface plasmons, which have frequency $\omega_{s I}$ in the local limit. The main aim of this paper is to show that an abrupt bimetallic interface does not support any hydrodynamic solution of surface plasmons.

\section{Dispersion relation}

The linearized equations governing the hydrodynamics and electrostatics of electron gas in a metal are:

$$
\frac{\partial n}{\partial t}+n_{0} \operatorname{div} v=0
$$




$$
\begin{aligned}
& \frac{\partial \mathbf{v}}{\partial t}=\frac{-e}{m} \mathbf{E}-\frac{\beta^{2}}{n_{0}} \nabla n, \\
& \nabla \cdot \mathbf{E}=-4 \pi e n, \\
& p=m \beta^{2} n,
\end{aligned}
$$

where $n$ is the electron density, $\mathrm{v}$ the velocity, $\boldsymbol{p}$ the pressure, $\mathbf{E}$ the electric field and $\beta^{2}=(3 / 5) v_{F}^{2}, v_{F}$ being the Fermi speed.

Considering the time dependence as $\exp (-i \omega t)$ and taking $E=-\operatorname{grad} \phi$, equations $(2)-(5)$ give

$$
\nabla^{4} \phi+k^{2} \nabla^{2} \phi=0
$$

where, $\quad k^{2}=\left(\omega^{2}-\omega_{p}^{2}\right) / \beta^{2}$,

and $\omega_{p}^{2}=4 \pi n_{0} e^{2} / m$ is the plasma frequency.

Taking the medium 1 to be $x<0$ and medium 2 to be $x>0$ lying in the plane $y-z$ the solutions of (6) for media 1 and 2 are; (the $y$ dependence is neglected and $z$-dependence along the interface is taken as $\exp ($ ilz $)$ ),

$$
\begin{aligned}
& \phi_{1}=A \exp \left(l^{2}-k_{1}^{2}\right)^{1 / 2} x+C \exp (l x), x<0, \\
& \phi_{2}=B \exp -\left(l^{2}-k_{2}^{2}\right)^{1 / 2} x+D \exp (-l x), x>0 .
\end{aligned}
$$

The electrostatic boundary conditions are: (i) $\phi$ must be continuous, (ii) $\partial \phi / \partial x$ must be continuous, (iii) The normal component of $j$, the current density i.e. $n_{0} e v_{x}$ must be continuous, and (iv) $\left(\beta^{2} / \omega_{p}^{2}\right) \mathrm{nm}$ must be continuous. The fourth boundary condition was derived by Forstmann and Stenschke (1977) from the continuity of the normal component of the energy current density.

From (4)

$$
n=\frac{1}{4 \pi e}\left[\frac{\mathrm{d}^{2} \phi}{\mathrm{d} x^{2}}-l^{2} \phi\right]
$$

and from (3) we get

$$
-i \omega v_{x}=\frac{e}{m}\left[\frac{\mathrm{d} \phi}{\mathrm{d} x}-\frac{\beta^{2}}{\omega_{p}^{2}}\left(\frac{\mathrm{d}^{3} \phi}{\mathrm{d} x^{3}}-l^{2} \phi\right)\right] .
$$

Applying the boundary conditions to the solutions (8) and $n$ and $v_{x}$ we get the dispersion relation:

$$
\left|\begin{array}{cccc}
1 & 1 & -1 & -1 \\
m_{1} & l & m_{2} & l \\
\frac{\omega^{2}-\omega_{p 1}^{2}}{\omega_{p 1}^{2}} & 0 & \frac{-\left(\omega^{2}-\omega_{p 2}^{2}\right)}{\omega_{p 2}^{2}} & 0 \\
\omega^{2} m_{1} & l \omega_{p 1}^{2} & \omega^{2} m_{2} & l \omega_{p 2}^{2}
\end{array}\right|=0 \text {, }
$$


where $\quad m_{1}^{2}=l^{2}-\frac{\omega^{2}-\omega_{p 1}^{2}}{\beta_{1}^{2}}$ and $m_{2}^{2}=l^{2}-\frac{\omega^{2}-\omega_{p 2}^{2}}{\beta_{2}^{2}}$.

\section{Discussion of the dispersion equation}

Equation (11) can be written as follows:

$$
\begin{gathered}
{\left[a^{2}-\left(\omega^{2}-1\right)\right]^{1 / 2}\left(\omega^{2}-\gamma\right)\left(\omega^{2}-\frac{1+\gamma}{2}\right)+\left[a^{2}-\left(\omega^{2}-\gamma\right) / \eta\right]^{1 / 2}} \\
\gamma\left(\omega^{2}-1\right)\left(\omega^{2}-\frac{1+\gamma}{2}\right)-\frac{(1-\gamma)^{2}}{2} a \omega^{2}=0,
\end{gathered}
$$

where $a=l \beta_{1} / \omega_{p 1}, \omega \equiv\left(\omega / \omega_{p 1}\right), \gamma=\omega_{p 2}^{2} / \omega_{p 1}^{2}$ and $\eta=\beta_{2}^{2} / \beta_{1}^{2}$ are the interface parameters.

We note that when $\gamma \rightarrow 0$, (since $\eta=0\left(\gamma^{4 / 3}\right)$ ), (12) reduces to

$$
\left[\alpha^{2}-\left(\omega^{2}-1\right)\right]^{1 / 2}\left(2 \omega^{2}-1\right)=\alpha,
$$

which is the same as that derived by Fuchs and Kliewer (1971; eq. (18)) for metalvacuum interface. Squaring both sides of (13) we get the exact expression for $\omega$ as

$$
\omega=\frac{a+\left(a^{2}+2\right)^{1 / 2}}{2}
$$

which for small a gives the well-known expression (1)

$$
\omega=\frac{\omega_{p 1}}{\sqrt{2}}\left[1+\left(\frac{3}{10}\right)^{1 / 2} \frac{F_{F} l}{\omega_{p 1}}\right]
$$

Now, we shall show that the dispersion equation (12) does not possess any real root $\alpha$, for a given value of $\omega$ except the roots $\omega^{2}=\gamma$ which represent the bulk modes in the two media. For this we shall write (12) as

$$
A(\omega)\left[\left(\alpha^{2}-\left(\omega^{2}-1\right)\right]^{1 / 2}+B(\omega)\left[\left(\alpha^{2}-\left(\omega^{2}-\gamma\right) / \eta\right)\right]=C(\omega) \alpha .\right.
$$

Since $A(\omega)$ and $B(\omega)$ change sign at $\omega^{2}=1, \omega^{2}=\gamma$ and $\omega^{2}=(1+\gamma) / 2$ we discuss the roots of (15) in the four regions (a) $0<\omega^{2}<\gamma$. (b) $\gamma<\omega^{2}<(1+\gamma) / 2$, (c) $(1+\gamma) / 2<\omega^{2}<1$ and (d) $1<\omega^{2}<\infty$. Without loss of generality we shall choose $\gamma<1$.

In region (a) it can easily be shown that $A(\omega)\left[\alpha^{2}+1-\omega^{2}\right]^{1 / 2}>C(\omega) a$, hence (15) is always positive. In region (b) we can show that $B(\omega)\left[\alpha^{2}-\left(\omega^{2}-\gamma\right) / \eta\right]^{1 / 2}-C(\omega) \alpha$ is negative, making (15) negative for all $a$. In region (c) we note that for real roots to exist the value of $a$ must be greater than $a_{\min }$, given by $a_{\min }=\left[\left(\omega^{2}-\gamma\right) / \eta\right]^{1 / 2}$. 
Hence taking into consideration that $a>a_{\min }$, we can show that $A(\omega)\left[\alpha^{2}-\left(\omega^{2}-1\right)\right]^{1 / 2}$ $\left\langle C(\omega) a\right.$ for all $a>a_{\min }$, thus making (15) negative for this range of $\omega^{2}$ also. In region (d) it can be easily shown that the asymptotes to the right side curves are greater than the straight line on left side, thus making (15) positive in this region of $\omega$.

\section{Conclusions}

The above analysis shows no roots for (12), hence there is no possibility of electrostatic wave propagation at the interface of two metals when hydrodynamic effects are taken into consideration. The only root admitted by (12) is $a=0, \omega^{2}=(1+\gamma) / 2$ which are the electrostatic oscillations when local dielectric model is taken into account. Hence, we note that in the presence of the second metal boundary the hydrodynamic correction, unlike the case when the medium is bounded by vacuum, does not introduce any dispersion to the electrostatic oscillations.

We would like to point out that in the above analysis we have chosen effective masses of the electrons in both the media to be equal. However, it is easy to show that the above conclusions remain valid even in the case when the effective masses are different.

Das Sarma and Quinn (1977) treated the problem of collective modes at a bimetallic junction including the effects of hydrodynamic dispersion. Though they do not specifically discuss the problem of non-existence of hydrodynamic solution for interface plasmons, they do state that in the case of abrupt bimetallic junction, dispersion relation obtained by standard boundary conditions (which differ from those in Forstmann and Stenschke 1977) turns out to be inconsistent with the assumption of a localized excitation whenever the compressibility of the electron gas is finite. This suggests that the non-existence of hydrodynamic solution for the interface plasmons is independent of the choice of the boundary conditions.

Doubts have been cast upon the applicability of the hydrodynamic approximation to the surface-plasmon problem (Harris 1971; Kleimann 1973). The discussions in this paper and the fact that the interfacial plasmon dispersion at a metallic surface has been calculated by Feibelman (1971) using the random-phase approximation (which gives the interface plasmon frequency $\omega_{s I}$ in the local limit), suggests that the use of usual hydrodynamic equation should be looked into carefully. The study of hydrodynamic solution of plasmons at the bimetallic interface employing an improved theory of hydrodynamics on similar lines as in Kleinman (1973) will form the topic for a separate paper.

\section{Acknowledgement}

Major part of this work was carried out when the first author (C Uberoi) was visiting the University of Autonoma de Puebla. The kind hospitality of Dr P Halevi and the partial financial support by CONACYT is gratefully acknowledged.

The authors thank Miss ElsaChavira for carrying out the preliminary numerical work. 


\section{References}

Das Sarma S and Quinn J J 1977 Bull. Am. Phys. Soc. 22537 Feibelman P J 1971 Phys. Rev. B3 2974

Forstmann F and Stenschke H 1977 Phys. Rev. Lett. 381365

Fuchs R and Kliewer K L 1971 Phys. Rev. B3 2270

Harris J 1971 Phys. Rev. B4 1022

Kleinman L 1973 Phys. Rev. B7 2288

Kunz C 1966 Z. Physik 196311

Stern E A and Ferrel R A 1960 Phys. Rev. 120130 